\title{
Application of Modified Spin-
} Echo-based Sequences for Hepatic MR Elastography: Evaluation, Comparison with the Conventional Gradient-Echo

\section{Sequence, and Preliminary Clinical} Experience $^{1}$

Yogesh K. Mariappan, PhD

Bogdan Dzyubak, PhD

Kevin J. Glaser, PhD

Sudhakar K. Venkatesh, MD

Claude B. Sirlin, MD

Jonathan Hooker, BS

Kiaran P. McGee, PhD

Richard L. Ehman, MD
${ }^{1}$ From the Department of Radiology, Mayo Clinic, 200 First St SW, Rochester, MN 55905 (Y.K.M., B.D., K.J.G., S.K.V., K.P.M., R.L.E.); and Department of Radiology, University of California, San Diego, Calif (C.B.S., J.H.) Received January 20, 2016; revision requested March 14; revision received May 2; accepted May 30; final version accepted June 13. Address correspondence to R.L.E. (e-mail: ehman.richard@mayo.edu).

Supported by National Institutes of Health grants EB001981, EB07593, and DK088925 and the Imaging Biomarker Discovery Program, Mayo Center for Individualized Medicine.

() RSNA, 2016
Purpose:

Materials and Methods:

Results:

Conclusion:
To (a) evaluate modified spin-echo (SE) magnetic resonance (MR) elastographic sequences for acquiring MR images with improved signal-to-noise ratio (SNR) in patients in whom the standard gradient-echo (GRE) MR elastographic sequence yields low hepatic signal intensity and (b) compare the stiffness values obtained with these sequences with those obtained with the conventional GRE sequence.

This HIPAA-compliant retrospective study was approved by the institutional review board; the requirement to obtain informed consent was waived. Data obtained with modified SE and SE echo-planar imaging (EPI) MR elastographic pulse sequences with short echo times were compared with those obtained with the conventional GRE MR elastographic sequence in two patient cohorts, one that exhibited adequate liver signal intensity and one that exhibited low liver signal intensity. Shear stiffness values obtained with the three sequences in 130 patients with successful GRE-based examinations were retrospectively tested for statistical equivalence by using a $5 \%$ margin. In 47 patients in whom GRE examinations were considered to have failed because of low SNR, the SNR and confidence level with the SE-based sequences were compared with those with the GRE sequence.

The results of this study helped confirm the equivalence of SE MR elastography and SE-EPI MR elastography to GRE MR elastography $(P=.0212$ and $P=.0001$, respectively). The SE and SE-EPI MR elastographic sequences provided substantially improved SNR and stiffness inversion confidence level in 47 patients in whom GRE MR elastography had failed.

Modified SE-based MR elastographic sequences provide higher SNR MR elastographic data and reliable stiffness measurements; thus, they enable quantification of stiffness in patients in whom the conventional GRE MR elastographic sequence failed owing to low signal intensity. The equivalence of the three sequences indicates that the current diagnostic thresholds are applicable to SE MR elastographic sequences for assessing liver fibrosis.

${ }^{\circ}$ RSNA, 2016 
M agnetic resonance (MR) elastography (1) is a noninvasive imaging technique that can spatially resolve the mechanical properties of soft tissues (2-9). Currently, the most important clinical application of MR elastography is for the assessment of hepatic fibrosis (10-13). In the U.S. Food and Drug Administrationapproved commercial implementation available from multiple manufacturers, referred to as the standard technique henceforth, shear waves at $60 \mathrm{~Hz}$ are introduced into the liver with a pneumatically activated driver and imaged with a phase-contrast gradient-echo (GRE) MR elastography sequence. The wave images are then processed to generate cross-sectional images that depict the tissue shear stiffness (elastograms) by using a mathematical inversion algorithm $(11,13)$. Sample results are shown in Figure 1.

GRE MR elastography is a robust technique, with a success rate of $94.4 \%$ in a large study of 1377

\section{Advances in Knowledge}

- Spin-echo (SE) and SE echo-planar imaging (EPI) hepatic MR elastographic pulse sequences with short echo times provide an adequate image signal-to-noise ratio (SNR) in patients in whom the current standard (gradientecho [GRE] MR elastography) fails because of low SNR, enabling measurement of shear stiffness in these patients.

- Acquisition with use of the modified SE and SE-EPI-based MR elastographic sequences yields stiffness values statistically equivalent to those calculated with the conventional GRE MR elastographic sequence (at a $\pm 5 \%$ margin), with $P$ values of .021 and .0001 , respectively.

- The equivalence of the three sequences suggests that the current clinical diagnostic threshold values established with GRE MR elastography can also be used to assess hepatic fibrosis with the modified SE sequences. patients (14). Among the technical failures, approximately $70 \%$ of cases can be attributed to low MR signal intensity within the liver (14), primarily owing to the deposition of iron that resulted in substantial transverse relaxation time as affected by magnetic field heterogeneity (T2*)-dependent signal reduction $(15,16)$ (Fig 1). This is an important clinical problem because iron deposition is a known coexistent condition in various chronic liver diseases $(17,18)$.

It was hypothesized that the liver MR signal intensity and, hence, the success rate of MR elastography can be improved by using pulse sequences that are less sensitive to $\mathrm{T} 2 *$ effects and that these sequences will provide stiffness measurements similar to those obtained with the standard clinical sequence. The goal of this study was to evaluate two spin-echo (SE)-based MR elastographic pulse sequences with short echo times (TEs) and to compare the data obtained with these sequences with those obtained by using the GRE MR elastographic sequence in situations in which the conventional technique had succeeded or failed.

\section{Materials and Methods}

The institutional review board approved the study, and the requirement to obtain written informed consent was waived for retrospective review of data in the patients who underwent clinically indicated MR elastography. This study was also compliant with the Health Insurance Portability and Accountability Act. Authors not associated with any

\section{Implication for Patient Gare}

- The SE and SE-EPI MR elastographic sequences can potentially be used to measure hepatic stiffness in patients in whom the GRE MR elastographic sequence may fail because of low SNR, increasing the technical success rate of MR elastography and thereby reducing the need for invasive liver biopsy. industry had control of all patient and volunteer data.

\section{Pulse Sequences}

The standard hepatic MR elastographic technique (13) uses a fast GRE pulse sequence with a bipolar motion-encoding gradient (Fig 2, A) lasting 16.66 msec that matches the wave duration of the $60-\mathrm{Hz}$ motion and has a TE of approximately $19.9 \mathrm{msec}$. This relatively long TE makes the sequence heavily $\mathrm{T} 2$ * weighted and sensitive to short $\mathrm{T} 2$ * species, such as iron-overloaded liver parenchyma, which can have a $\mathrm{T}^{*}$ value as low as $1.3 \mathrm{msec}(15)$. Thus, the challenge is to acquire an MR signal intensity with sufficiently high signal-tonoise ratio $(\mathrm{SNR})$ to resolve the shear wave motion encoded into the phase of the MR signal intensity. Toward this end, two SE-based pulse sequences (that are inherently $\mathrm{T} 2 *$ insensitive because of the $180^{\circ}$ refocusing pulse) with short TEs owing to improvements similar to pulmonary MR elastographic sequences, as described by Mariappan et al (19), were evaluated in this study.

In one implementation, a single-echo SE MR elastographic pulse sequence with such modifications as fractional motion encoding and crusher gradient removal $(19,20)$ was used. The sequence

\section{Published online before prin \\ Radiology 2017; 282:390-398 \\ Abbreviations: \\ $\mathrm{EPI}=$ echo planar imaging \\ $\mathrm{GRE}=$ gradient echo \\ $\mathrm{SE}=$ spin echo \\ SNR $=$ signal-to-noise ratio \\ $\mathrm{TE}=$ echo time} 10.1148/radiol.2016160153 Content codes: GI MR

\section{Author contributions:}

Guarantors of integrity of entire study, Y.K.M., B.D.; study concepts/study design or data acquisition or data analysis/ interpretation, all authors; manuscript drafting or manuscript revision for important intellectual content, all authors manuscript final version approval; agrees to ensure any questions related to the work are appropriately resolved, all authors; literature research, Y.K.M., K.J.G., R.L.E.; clinical studies, Y.K.M., S.K.V., R.L.E.; statistical analysis, B.D., R.L.E.; and manuscript editing, Y.K.M., B.D., K.J.G., S.K.V., C.B.S., K.P.M., R.L.E.

Conflicts of interest are listed at the end of this article. 
Figure 1
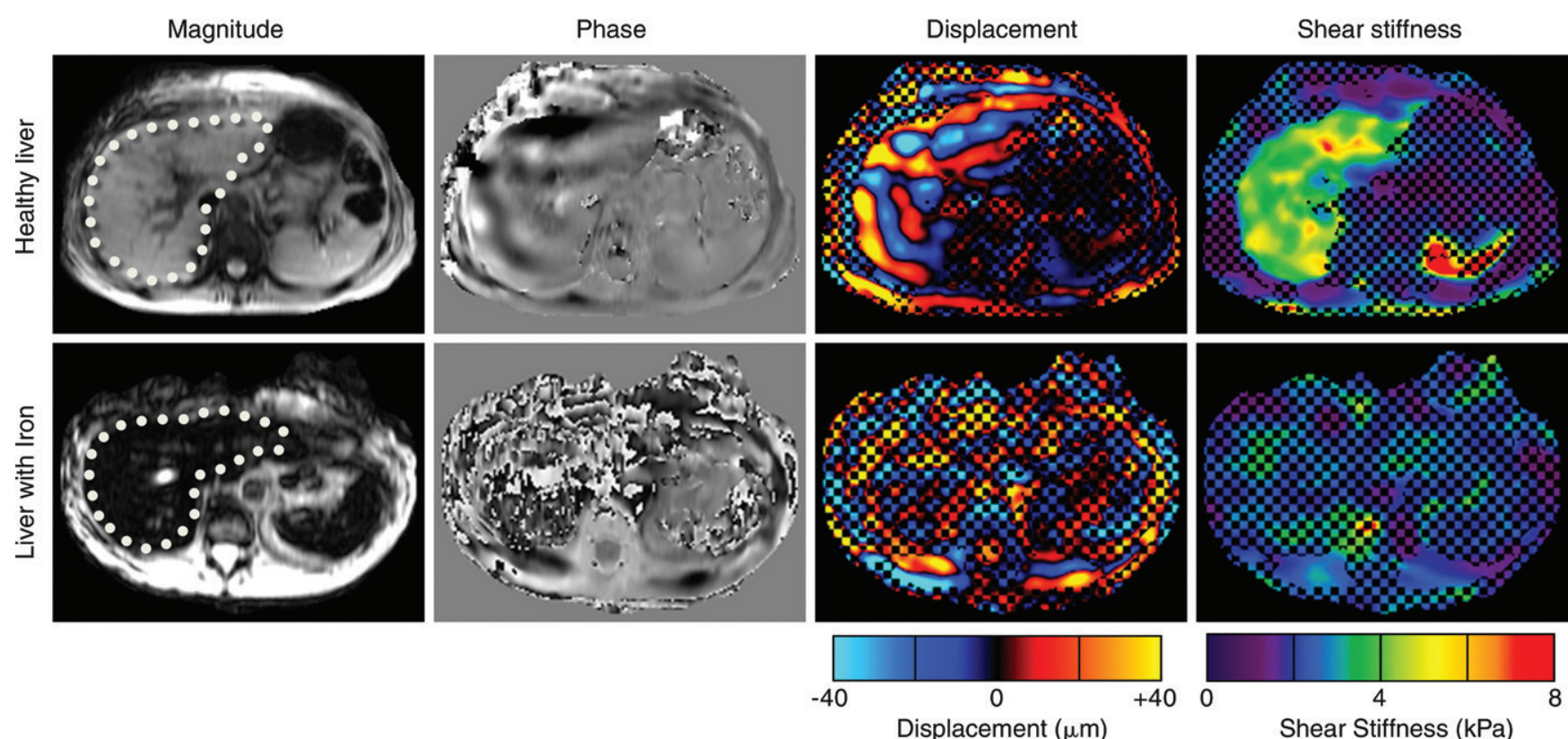

Figure 1: MR elastographic images in patients in whom conventional GRE MR elastography was successful (top row) or failed (owing to liver iron overload) (bottom row). Liver is indicated with dotted line. In a typical MR elastography case (top row), magnitude signal intensity is high, shear waves are well visualized on phase and displacement images, and shear stiffness can be successfully calculated within liver on derived stiffness image. In images from the patient with low signal intensity in liver (bottom row), visualization of waves is not possible on phase and displacement images, which results in invalid shear stiffness results. Checkerboard pattern superimposed on displacement and stiffness images represents a mask applied by processing algorithm to areas where phase SNR is below a value that permits reliable evaluation of stiffness.

\section{Figure 2}
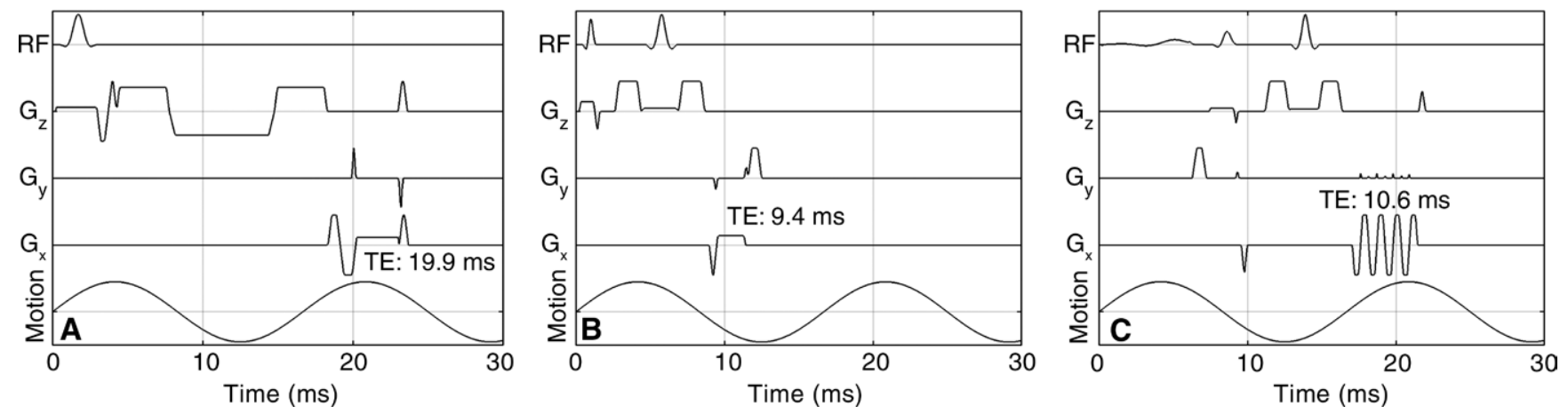

Figure 2: Three pulse sequences investigated in this study. $A$, Conventional GRE MR elastographic sequence. $B$, SE MR elastographic sequence. $C$, SE-EPI MR elastographic sequence. TE for each of the three sequences and a schematic of the $60-\mathrm{Hz}$ motion are also shown. Radiofrequency (RF) row indicates the radiofrequency application (excitation and inversion), $G_{x^{\prime}} G_{\gamma^{\prime}} G_{z}$ are MR imaging spatial-encoding and motion-encoding gradients, and "Motion" refers to mechanical vibrations introduced by using the MR elastography acoustic driver.

(Fig 2, B) included two unipolar motionencoding gradients, each $2 \mathrm{msec}$ in $\mathrm{du}^{-}$ ration, and a TE of 9.4 msec. Although these modifications decrease the TE, this results in a reduction in shear-wave motion sensitivity (19). For instance, the SE MR elastographic sequence has a sensitivity of $52 \mu \mathrm{m} / \mathrm{rad}$ for $60-\mathrm{Hz}$ vibrations (ie, every radian of phase accumulation needs $52 \mu \mathrm{m}$ of motion), whereas the GRE MR elastographic sequence has a sensitivity of $10 \mu \mathrm{m} / \mathrm{rad}$.
In addition to the SE MR elastography sequence, we evaluated a SE echoplanar imaging (EPI) MR elastographic sequence $(19,21)$ with modifications similar to those of the SE MR elastographic pulse sequence, which allows 
for faster imaging. This sequence allowed the total acquisition time and the number of breath holds to be reduced by half compared with the GRE MR elastographic and SE MR elastographic sequences. The sequence (Fig 2, C) had an echo train length of eight and a TE of approximately $10.6 \mathrm{msec}$. The motion sensitivity was $62 \mu \mathrm{m} / \mathrm{rad}$.

\section{Data Acquisition and Analysis}

All experiments were performed by using 1.5-T whole-body MR imaging units (GE Healthcare, Waukesha, Wis) with standard torso coil arrays. Continuous $60-\mathrm{Hz}$ vibrations were introduced into the liver, and MR elastographic data were acquired with the three sequences by using these common parameters: four 10-mm axial sections, 42-46-cm field of view, superior-inferior motion-encoding gradients, $256 \times 256$ reconstruction matrix, and four phase offsets. GRE MR elastography was performed with four breath holds of 15 seconds each, a 50msec repetition time, a parallel imaging factor of 2 , and a $256 \times 64$ acquisition matrix. SE MR elastography was performed with four 18-second breath holds, a 200-msec repetition time, a $128 \times$ 64 acquisition matrix, no parallel imaging, and $0.6-0.8$ fractional phase field of view. SE-EPI MR elastography was performed with two 13-second breath holds, a 330-msec repetition time, a parallel imaging factor of 2 , and an $80 \times 80$ acquisition matrix. Both the SE and SEEPI MR elastographic sequences (collectively called the SE sequences hereafter) had a $50 \%$ increase in the amplitude of motion of the pneumatic driver system compared with GRE MR elastographic acquisition to compensate for the reduced motion sensitivity.

The standard clinical MR elastographic inversion algorithm, with a direct inversion of the Helmholtz wave equation (22) and spatiotemporal directional filters (23), was used to calculate the shear stiffness maps. The inversion algorithm reports a confidence map based on the correlation coefficients of polynomial fits to the wave data. A threshold value of 0.95 was used to differentiate between regions of reliable and unreliable shear wave data, as is standard clinical practice. A checkerboard pattern is added to the displacement and the shear stiffness images to indicate the regions where the stiffness estimation is unreliable. Mean liver stiffness was calculated by using an automated segmentation and region of interest-generation algorithm (24) and is reported in units of kilopascals. The MR elastographic examination was considered successful if the region of interest contained at least 500 pixels with reliable stiffness values within the liver in each section (25).

\section{Patient Study}

The modified SE sequences were included in the routine clinical imaging protocol for patients indicated for MR elastography at our institution. We selected two cohorts of patients in this study: one in which the conventional GRE MR elastographic sequences were technically successful and another in which those sequences failed because of low-magnitude signal intensity.

Because the wave propagation physics remains the same, the new pulse sequences should provide stiffness measurements equivalent to those obtained with the current clinical standard of reference. To assess this, an equivalence study was performed by retrieving MR elastographic data in consecutive patients imaged between March and June 2013 from the clinical archive. The data were reviewed by a researcher (B.D.) with 3 years of experience with MR elastography; of the 165 patients used for the stiffness validation part of the study, 29 were excluded because of failure in one or more of the three acquisition sequences. Of these 29 patients, 18 patients were excluded because of driver disconnection and/or poor coupling, four patients had severe artifacts from patient motion (three in all sequences, one only in the SE sequence), three patients had motion between the MR elastographic phases (SE and SE-EPI only), three patients had examinations with low motion amplitude (in SE and SE-EPI sequences), and one patient had an incorrectly prescribed section. A further six patients were excluded because of segmentation failures, resulting in a total of 130 patients who had successful acquisitions with all three sequences. This patient group had an age range of 19-81 years (mean, 51 years) and included 59 women. The mean ages for men and women were 52 years (range, 19-80 years) and 55.8 years (range, 22-81 years), respectively. The difference in ages was not significant $(P=.11)$.

In the next part of the study, we evaluated the ability of the new pulse sequences to improve data quality in patients with low signal intensity at GRE MR elastography. The direct relationship between image SNR and inversion quality is unknown, so low-signal-intensity examinations were defined as those in which less than $30 \%$ of the imaged liver volume, calculated by using the automated segmentation, had an MR elastographic inversion confidence level of more than 0.95, which is the current standard confidence level threshold used to mask out examinations for the stiffness measurement. Fifty-two such examinations from 2013 to 2015 that also had SE and SE-EPI acquisitions were retrieved from the clinical archive. These data were screened to exclude examinations that had low confidence because of acoustic driver problems (five examinations), and this resulted in 47 examinations that had SE and SE-EPI acquisitions as well as a failed $(<30 \%$ of the liver with high confidence) GRE acquisition. The livers in these examinations were segmented by using the automated approach, and the following parameters were compared among the GRE, SE, and SE-EPI sequences: (a) SNR (mean value within the liver/standard deviation within the liver), (b) mean confidence level within the liver, and (c) fraction of the liver with an MR elastographic confidence level higher than 0.95. The SNR metric was chosen because measurements in the background pixels may not be reliable as a result of masking in array coil spatial sensitivity encodingaccelerated images as well as ghosting in EPI and SE acquisitions.

\section{Statistical Analysis}

Stiffness values were calculated from automated regions of interest in successful 
Figure 3
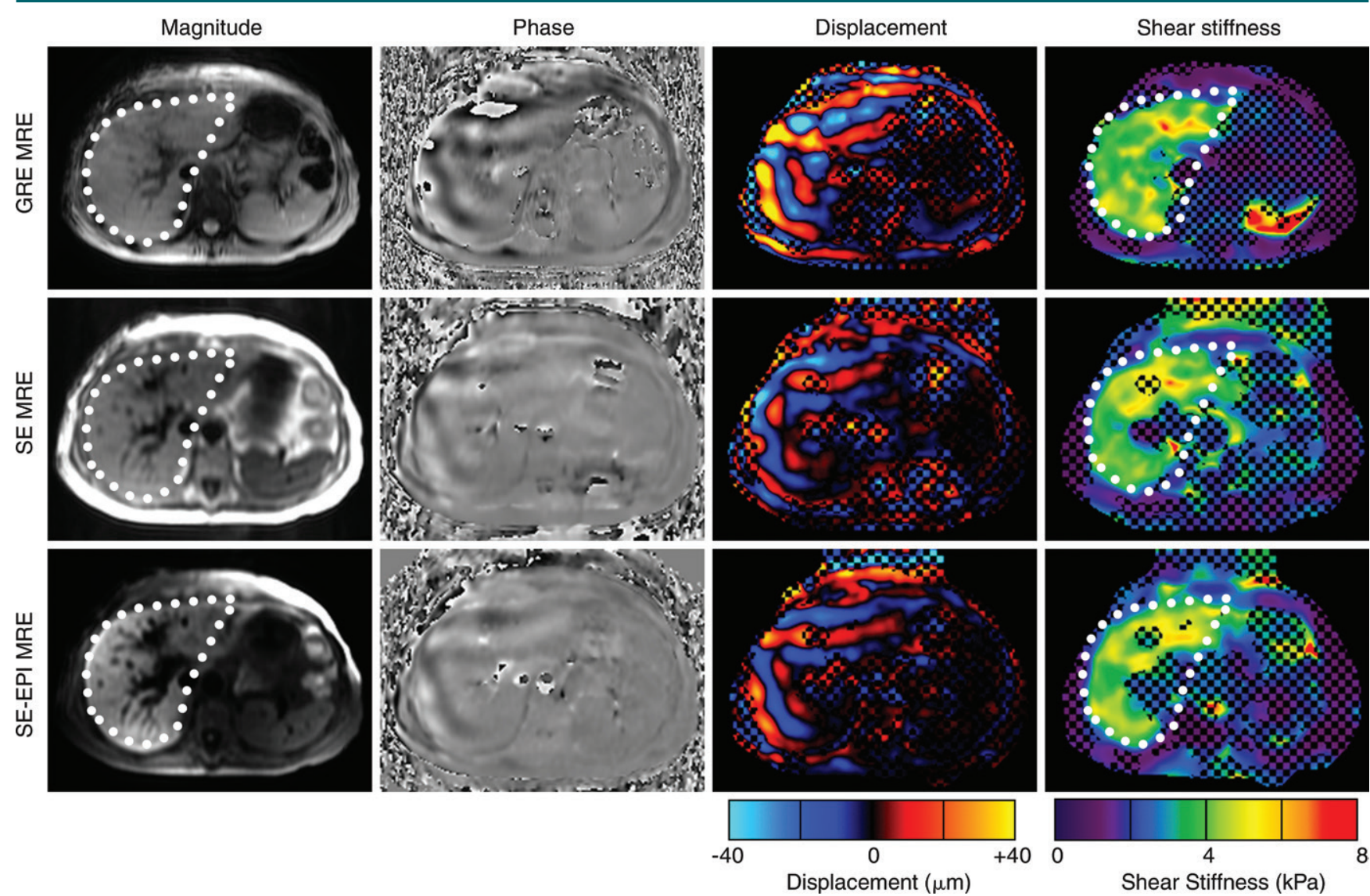

Figure 3: MR elastographic (MRE) images obtained in patient by using GRE MR elastographic (top row), SE MR elastographic (middle row), and SE-EPI MR elastographic (bottom row) techniques. Shear stiffness maps obtained with the three sequences are similar, demonstrating the equivalence of these three techniques. Checkerboard pattern on elastograms indicates regions where phase SNR is low (inversion confidence $<0.95$ ). Liver is indicated with dotted line.

SE and SE-EPI sequences $(>0.3$ of the liver with $>0.95$ confidence). For the first part of the study, stiffness values were calculated from automated regions of interest with all three sequences, and a paired statistical equivalence test (26) was used to compare the stiffness values obtained from SE and SE-EPI with values obtained with the clinical GRE technique; the commercially available software package JMP was used (JMP 9.0; SAS Institute, Cary, NC). The test had the following hypotheses: HO, the absolute mean stiffness differences with GRE are greater than the equivalence margin, e, and, H1, the absolute mean stiffness differences with GRE are less than $e$.

The equivalence margin, which indicates the smallest practical difference, was set to $5 \%$. The selection was based on the fact that experienced readers of MR elastographic images have a reproducibility of $\pm 10 \%$ (25), so a change in stiffness of $5 \%$ is not clinically significant. The significance $(\alpha)$ value was set at .05. This setup of hypotheses, which is opposite to the setup of the more common test for difference, allows the error from the low sample size to be controlled. Thus, a low $P$ value obtained with an equivalence test supports similarity of the distributions, whereas a high $P$ value obtained from a test for differences can only provide weak support because it can result from similarity of distributions or a small sample size. The stiffness values were also compared by using BlandAltman analysis. For the second part of the study, the SNRs, average confidence levels, and high-confidence areas obtained with the three pulse sequences were compared by using a paired $t$ test, with the $P$ value significance threshold set to .05 .

\section{Results}

Example data sets obtained with the three sequences in a patient with a successful GRE MR elastographic sequence are shown in Figure 3. The shear stiffness maps obtained with the SE sequences were similar to the data obtained with the GRE MR elastographic technique. The mean stiffness values ( \pm standard deviation) obtained with the GRE, SE, and SE-EPI MR elastographic sequences in the 130-patient data set 


\section{Figure 4}
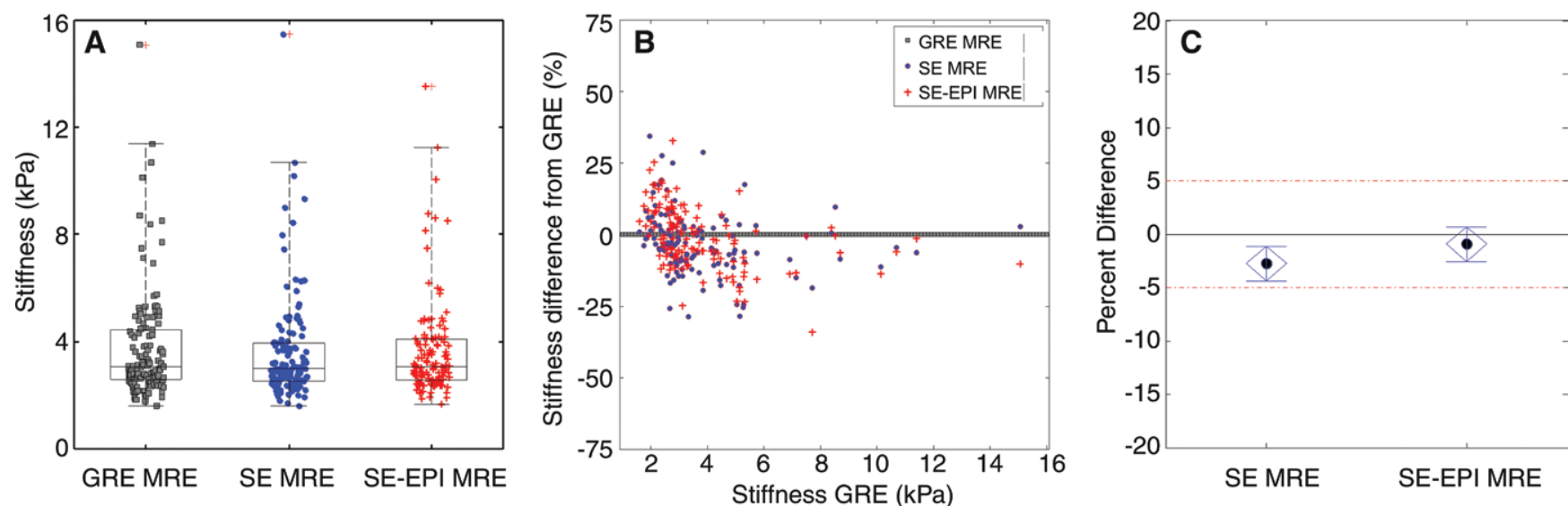

Figure 4: $A$, Comparison of shear stiffness values in 130 patients with the three MR elastographic (MRE) sequences. Box-and-whisker plot shows median, first, and third quartile range, and 99th percentile. B, Bland-Altman plot of percentage differences of shear stiffness values obtained with SE and SE-EPI MR elastography (MRE) compared with GRE MR elastography. C, Mean percentage difference between stiffness from SE and SE-EPI sequences compared with GRE data. Confidence intervals for mean percentage differences were $-4.35 \%,-1.14 \%$, and $-2.52 \%, 0.65 \%$, respectively, and are shown with blue diamonds. Both intervals were fully within the $5 \%$ equivalence range, which indicates that the three sequences are statistically equivalent.

were $4.1 \mathrm{kPa} \pm 0.94,3.9 \mathrm{kPa} \pm 0.97$, and $4.0 \mathrm{kPa} \pm 1.0$, respectively. Figure 4 shows comparisons of individual and mean stiffness values obtained with the three sequences. The mean 95\% Bland-Altman confidence interval for the percentage differences in stiffness, shown in Figure $4, B$, was $2.1 \% \pm 21.4$ for the $\mathrm{SE}$ sequence and $0.9 \% \pm 15.4$ for the SE-EPI sequence. The paired equivalence test rejected the null in favor of the alternative hypothesis that the SE and SE-EPI MR elastographic sequences were equivalent to the GRE MR elastographic sequence, with $P$ values of .0212 and .0001 , respectively.

Figure 5 shows images from one such patient in whom the GRE acquisition had failed. The SE sequences provided sufficient signal intensity, with an average confidence level of more than 0.9 , compared with an average confidence level of less than 0.3 with GRE MR elastography, allowing liver stiffness to be reliably estimated. The stiffness result was reported clinically on the basis of the SE sequence.

In low-signal-intensity examinations in which less than $30 \%$ of the liver had high inversion confidence at GRE MR elastography (and hence were considered to have failed), the average confidence, confidence area, and SNR were all improved with the new pulse sequences $(P<.0001)$, as can be seen in the Table. This improvement brought the data quality close to the levels of the GRE examinations with normal results in the 130-patient data set, for whom the area with high confidence, average confidence, and SNR were $69.7 \%, 0.944$, and 4.97 , respectively. The SE and SE-EPI sequences yielded successful examinations, with more than $30 \%$ high confidence liver area in $43(91.5 \%)$ and $44(93.6 \%)$ of the 47 examinations, respectively. Both SE and SE-EPI sequences were performed successfully in 42 of the 47 examinations and yielded statistically equivalent stiffness values $($ mean $=3.99 \mathrm{kPa}$ with the SE sequence and $3.42 \mathrm{kPa}$ with the SE-EPI sequence; $P<.001$ ). Figure 6 shows the correlation of stiffness values obtained with the two modified SE sequences. On the basis of the stiffness values obtained with the $\mathrm{SE}$ sequences, 14 of 47 patients had stiffness values in the abnormal range, thereby indirectly supporting their clinical utility.

\section{Discussion}

MR elastography has been established as a noninvasive diagnostic tool for the assessment of hepatic fibrosis.
Currently, a fast GRE-based MR elastographic sequence is the most widely used technique. However, the heavy T2* weighting provided with this sequence makes it unsuitable for patients with low $\mathrm{T}^{*}$ values in the liver (eg, patients with iron overload), which results in low liver signal intensity. Although previous studies have investigated the use of SE MR elastographic sequences, these sequences have failed in these types of patients because of long TEs (27). To quantify hepatic stiffness in these patients, two SE-based sequences with short TEs that are less sensitive to T2*, which were developed for pulmonary MR elastography (19), were evaluated in patients with typical and low SNR on the GRE MR elastographic images.

Modified SE and SE-EPI MR elastographic pulse sequences provide measurements of shear stiffness equivalent to those obtained with the current clinical standard and provide adequate image SNR in patients in whom the conventional technique has failed because of low-magnitude SNR. Thus, modified SE and SE-EPI MR elastography may allow assessment of hepatic fibrosis in these patients. The observed agreement among the three sequences was better than or similar to the interreader agreement for the stiffness calculation 

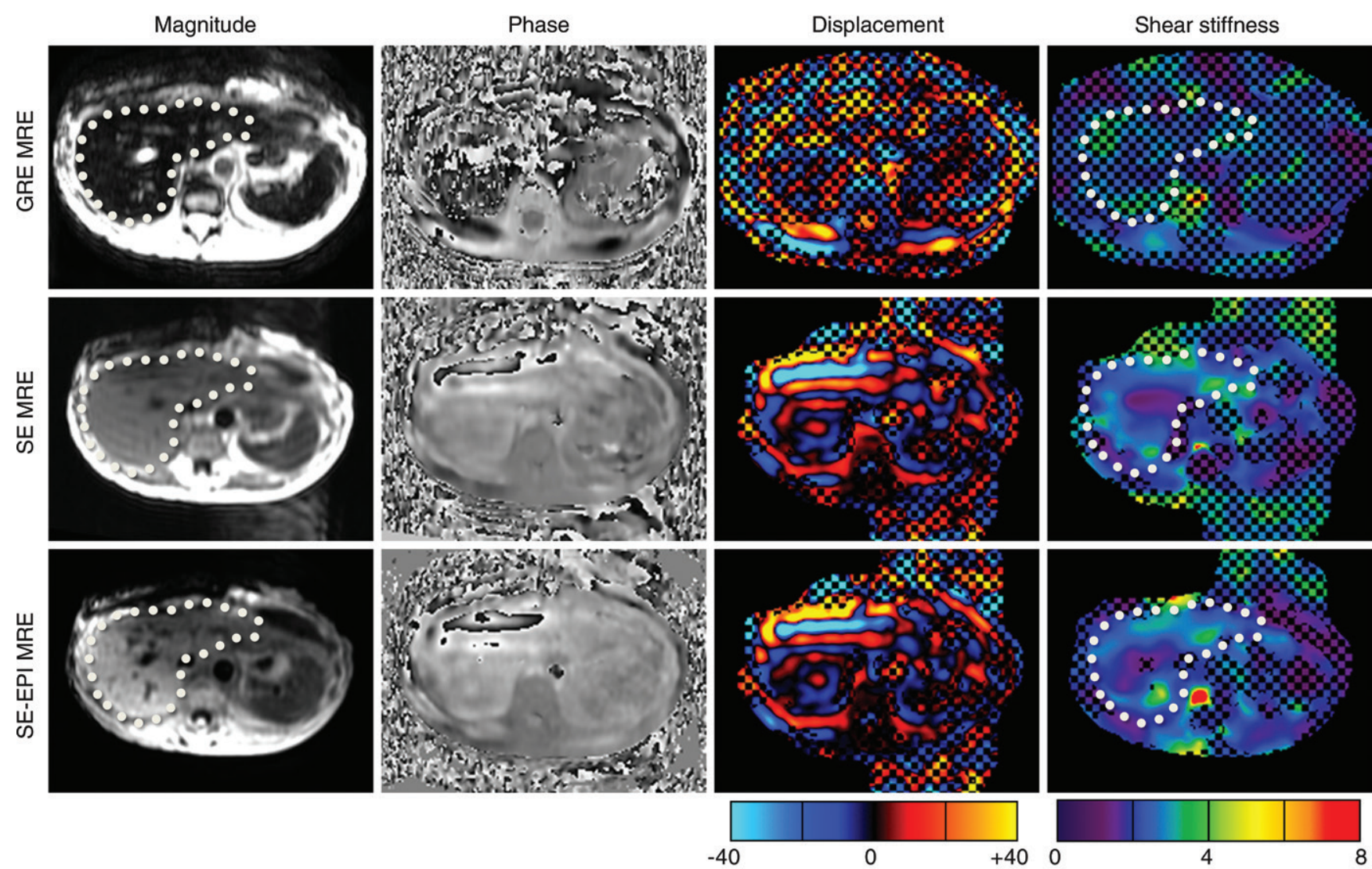

Displacement $(\mu \mathrm{m})$

Shear Stiffness $(\mathrm{kPa})$

Figure 5: MR elastographic (MRE) images in patient with low signal intensity at GRE MR elastography. Images were obtained with GRE MR elastography (top row), SE MR elastography (middle row), and SE-EPI MR elastography (bottom row). Liver is indicated with dotted line. For GRE MR elastography, signal intensity level in liver was low and resulted in noise-dominated phase images and a stiffness map fully masked out because of low inversion confidence. Conversely, both SE sequences had significantly improved SNR and confidence level, with waves clearly visualized on phase-contrast images. Mean hepatic stiffness values were $2.3 \mathrm{kPa} \pm 0.83$ for SE MR elastography and $2.4 \mathrm{kPa} \pm 0.77$ for SE-EPI MR elastography; the GRE data were considered unusable.

from MR elastographic images $(25,28)$. Hence, it is likely that these sequences may be used for the clinical assessment of hepatic fibrosis by using the current diagnostic thresholds derived from previous studies with the GRE MR elastographic sequence $(11,13)$.

In the preliminary clinical data reported herein, the SE and SE-EPI MR elastographic methods were successful in the cohort of 47 patients, indicating fibrosis in 15 patients. SE sequences thus provide viable options for measuring the stiffness of livers when the conventional sequence fails as a result of low-magnitude image SNR, obviating invasive biopsies. A failure owing to low SNR at conventional GRE MR

\begin{tabular}{|c|c|c|c|}
\hline \multicolumn{4}{|c|}{ Comparison of Data Quality in 47 Patients with Low Signal Intensity at GRE } \\
\hline Variable & GRE Sequence & SE Sequence & SE-EPI Sequence \\
\hline Area with high confidence $(\%)$ & 9.1 & 67.1 & 66.1 \\
\hline Average confidence & 0.629 & 0.946 & 0.943 \\
\hline SNR & 1.344 & 3.475 & 3.452 \\
\hline
\end{tabular}

elastography is easily assessed by reviewing the images obtained during the MR elastographic sequence at the imaging unit; an SE or SE-EPI sequence can be performed to obtain technically successful MR elastographic images. The new SE variants of the acquisition may allow the relationship among stiffness, fibrosis, and iron overload to be studied directly, as has been done with ultrasonography (25). Until now, this has not been possible with MR elastography. However, because the level of iron deposition can be high in diseases such as hemochromatosis and thalassemia, further research is needed to determine 
Figure 6

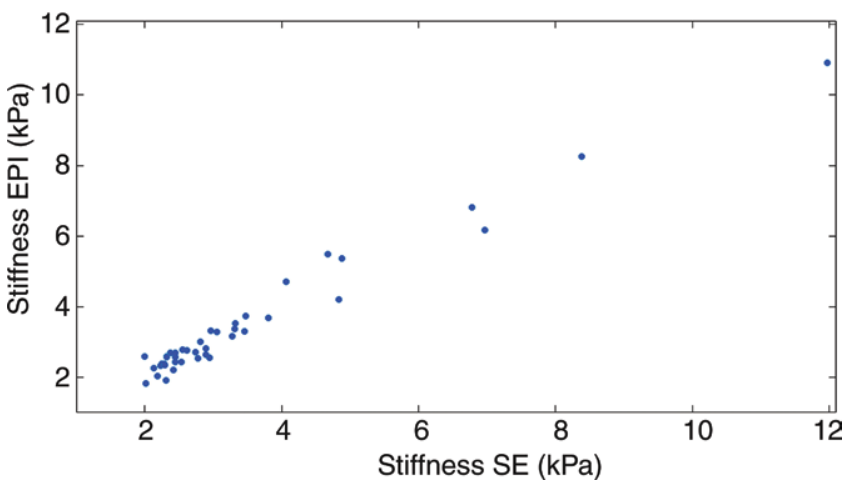

Figure 6: Shear stiffness values obtained with SE and SE-EPI sequences in 47 patients with nonanalyzable GRE MR elastographic images. Stiffness values calculated with the two sequences are equivalent $(P<.001)$, which suggests that the current threshold for fibrosis detection can be used for data obtained with the new acquisitions. According to this threshold, 14 of 47 patients had fibrosis.

the $\mathrm{T}^{*}$ threshold at which the SE variants may fail.

The use of SE-based MR elastographic pulse sequences has many limitations. First, the motion sensitivity of these modified SE sequences is lower than that with the conventional GRE MR elastographic sequence. This can be partially compensated for by increasing the motion amplitude of the acoustic vibrations at the source because the driver is typically run at less than $50 \%$ of maximum amplitude. Second, because of the use of unipolar motion-encoding gradients, the SE sequences are more sensitive to flow and cardiac motion artifacts. Third, long repetition times are desirable to allow for sufficient longitudinal relaxation for the SE sequences, which can extend the breath-hold times. In the GRE MR elastographic acquisition, all of the time offsets for one section are collected in one breath hold. However, this is not as practical with the SE acquisitions, and the time offsets are split over multiple breath holds. This may result in potential misregistration issues, especially in patients who are poor breath holders. However, because multiple sections of data can be acquired within a repetition time with the SE sequences, the total acquisition times for both the GRE and SE MR elastographic sequences to acquire four phase-offset images for four sections are similar. With the SEEPI MR elastographic sequence, because the data are acquired in approximately half the time and with half the number of breath holds compared with SE MR elastographic acquisition, the breath hold reproducibility artifacts are less pronounced.

Another limitation of this study is that low GRE signal intensity was defined by using inversion confidence. Lack of acoustic vibration delivered to the liver (eg, owing to driver malfunction) can also result in low confidence. The difference between the two causes is apparent visually, however, and with the new sequences available both issues can be resolved directly at the imaging unit.

In conclusion, our results show that the SE and SE-EPI MR elastographic sequences evaluated in this study can help quantify hepatic shear stiffness in patients in whom the current clinical MR elastographic technique fails because of image SNR of insufficient magnitude. These modified MR elastographic sequences provide shear stiffness measurements that are equivalent to those obtained with the standard MR elastographic sequence that is currently available, allowing current clinical diagnostic threshold values to be used.
Disclosures of Conflicts of Interest: Y.K.M. Activities related to the present article: disclosed no relevant relationships. Activities not related to the present article: is employed by Philips Healthcare. Other relationships: disclosed no relevant relationships. B.D. disclosed no relevant relationships. K.J.G. Activities related to the present article: disclosed no relevant relationships. Activities not related to the present article: has stock/stock options in Resoundant. Other relationships: disclosed no relevant relationships. S.K.V. disclosed no relevant relationships. C.B.S. Activities related to the present article: disclosed no relevant relationships. Activities not related to the present article: performed contracted work through the university for Alexion, Bioclinica, Biomedical System, Bristo Meyers Squibb, Galmed, GE, Genzyme, Gilead, Intercept Pharmaceuticals, Isis, Janssen, NuSirt, Pfizer, Profil, Sanofi, Synageva, Takeda, Virtualscopics, and AstraZeneca; received a grant from GE, Guerbet, and Siemens; is a paid consultant for Fibrogen, Tobira, and Virtualscopics; has a confidentiality and disclosure agreement with Icon, Intercept Pharmaceuti cals, Perspectum, and Shire to discuss contracted work through the university. Other relationships: disclosed no relevant relationships. J.H. disclosed no relevant relationships. K.P.M. Activities related to the present article: disclosed no relevant relationships. Activities not related to the present article: is a shareholder with Resoundant. Other relationships: disclosed no relevant relationships. R.L.E. Activities related to the present article: disclosed no relevant relationships. Activities not related to the present article: is the CEO of Resoundant; received a grant from Resoundant. Other relationships: has MR elastography patents issued and licensed to Resoundant; has royalties to MR elastography patents issued and licensed to Resoundant.

\section{References}

1. Muthupillai R, Lomas DJ, Rossman PJ, Greenleaf JF, Manduca A, Ehman RL. Magnetic resonance elastography by direct visualization of propagating acoustic strain waves. Science 1995;269(5232):1854-1857.

2. Mariappan YK, Glaser KJ, Ehman RL. Magnetic resonance elastography: a review. Clin Anat 2010;23(5):497-511.

3. Sack I, Beierbach B, Hamhaber U, Klatt D, Braun J. Non-invasive measurement of brain viscoelasticity using magnetic resonance elastography. NMR Biomed 2008;21(3): 265-271.

4. Sinkus R, Tanter M, Xydeas T, Catheline S, Bercoff J, Fink M. Viscoelastic shear properties of in vivo breast lesions measured by MR elastography. Magn Reson Imaging 2005; 23(2):159-165.

5. Elgeti T, Beling M, Hamm B, Braun J, Sack I. Cardiac magnetic resonance elastography: toward the diagnosis of abnormal myocardial relaxation. Invest Radiol 2010;45(12): $782-787$. 
6. Wuerfel J, Paul F, Beierbach B, et al. MRelastography reveals degradation of tissue integrity in multiple sclerosis. Neuroimage 2010;49(3):2520-2525.

7. Sahebjavaher RS, Baghani A, Honarvar M, Sinkus R, Salcudean SE. Transperineal prostate MR elastography: initial in vivo results. Magn Reson Med 2013;69(2):411-420.

8. Mariappan YK, Glaser KJ, Hubmayr RD, Manduca A, Ehman RL, McGee KP. MR elastography of human lung parenchyma: technical development, theoretical modeling and in vivo validation. J Magn Reson Imaging 2011;33(6):1351-1361.

9. Green MA, Sinkus R, Gandevia SC, Herbert RD, Bilston LE. Measuring changes in muscle stiffness after eccentric exercise using elastography. NMR Biomed 2012;25(6):852-858.

10. Asbach P, Klatt D, Schlosser B, et al. Viscoelasticity-based staging of hepatic fibrosis with multifrequency MR elastography. Radiology 2010;257(1):80-86.

11. Yin M, Talwalkar JA, Glaser KJ, et al. Assessment of hepatic fibrosis with magnetic resonance elastography. Clin Gastroenterol Hepatol 2007;5(10):1207-1213.e2.

12. Huwart L, Sempoux C, Vicaut E, et al. Magnetic resonance elastography for the noninvasive staging of liver fibrosis. Gastroenterology 2008;135(1):32-40.

13. Venkatesh SK, Yin M, Talwalkar JA, Ehman RL. Application of liver MR elastography in clinical practice [abstr]. In: Proceedings of the Sixteenth Meeting of the International
Society for Magnetic Resonance in Medicine. Berkeley, Calif: International Society for Magnetic Resonance in Medicine, 2008; 2611.

14. Yin M, Talwalkar J, Glaser K, Ehman RL. MR elastography of the liver: observations from a review of 1,377 exams [abstr]. In: Proceedings of the Nineteenth Meeting of the International Society for Magnetic Resonance in Medicine. Berkeley, Calif: International Society for Magnetic Resonance in Medicine, 2011; 390.

15. Sirlin CB, Reeder SB. Magnetic resonance imaging quantification of liver iron. Magn Reson Imaging Clin N Am 2010;18(3):359381 , ix.

16. Kaltwasser JP, Gottschalk R, Schalk KP, Hartl W. Non-invasive quantitation of liver iron-overload by magnetic resonance imaging. Br J Haematol 1990;74(3):360-363.

17. Pietrangelo A, Caleffi A, Corradini E. NonHFE hepatic iron overload. Semin Liver Dis $2011 ; 31(3): 302-318$.

18. Fargion S, Valenti L, Fracanzani AL. Beyond hereditary hemochromatosis: new insights into the relationship between iron overload and chronic liver diseases. Dig Liver Dis 2011;43(2):89-95.

19. Mariappan YK, Glaser KJ, Levin DL, et al. Estimation of the absolute shear stiffness of human lung parenchyma using (1) H spin echo, echo planar MR elastography. J Magn Reson Imaging 2014;40(5):1230-1237.

20. Rump J, Klatt D, Braun J, Warmuth C, Sack I. Fractional encoding of harmonic motions in MR elastography. Magn Reson Med 2007; 57(2):388-395

21. Edelman RR, Wielopolski P, Schmitt F. Echo-planar MR imaging. Radiology 1994;192(3):600-612.

22. Manduca A, Oliphant TE, Dresner MA, et al. Magnetic resonance elastography: noninvasive mapping of tissue elasticity. Med Image Anal 2001;5(4):237-254.

23. Manduca A, Lake DS, Kruse SA, Ehman RL. Spatio-temporal directional filtering for improved inversion of MR elastography images. Med Image Anal 2003;7(4):465-473.

24. Dzyubak B, Venkatesh SK, Manduca A, Glaser KJ, Ehman RL. Automated liver elasticity calculation for MR elastography. J Magn Reson Imaging 2016;43(5):1055-1063.

25. Dzyubak B, Glaser K, Yin M, et al. Automated liver stiffness measurements with magnetic resonance elastography. J Magn Reson Imaging 2013;38(2):371-379.

26. Dasgupta A, Lawson KA, Wilson JP. Evaluating equivalence and noninferiority trials. Am J Health Syst Pharm 2010;67(16): 1337-1343.

27. Bohte AE, van Dussen L, Akkerman EM, et al. Liver fibrosis in type I Gaucher disease: magnetic resonance imaging, transient elastography and parameters of iron storage. PLoS One 2013;8(3):e57507.

28. Shire NJ, Yin M, Chen J, et al. Test-retest repeatability of MR elastography for noninvasive liver fibrosis assessment in hepatitis C. J Magn Reson Imaging 2011;34(4):947-955. 